\title{
Antibioticoprofilaxia com Ampicilina na Rotura Prematura das Membranas. Estudo Randomizado e Duplo Cego
}

Ampicillin Prophylaxis in Premature Rupture of Membranes. Randomized and Double-Blind Study

José Elias Soares da Rocha, Geraldo Duarte, Sérgio Pereira da Cunha

Antonio Alberto Nogueira, Franciso Mauad Filho

\begin{abstract}
RESUMO
Objetivos: avaliar se o uso profilático da ampicilina pode evitar ou reduzir a morbidade infecciosa materna e perinatal decorrente da ruptura prematura das membranas (RPM) $e$ prolongar a gestação em pacientes portadoras dessa complicação.

Métodos: estudo prospectivo, randomizado e duplo-cego, avaliando 121 gestantes portadoras de RPM, divididas em dois grupos. O grupo tratado (61 gestantes) recebeu ampicilina e o grupo controle (60 gestantes) recebeu placebo, nas mesmas condições de horário, tempo de uso, embalagem e cor das cápsulas. Como parâmetros de infecção materna considerou-se a morbidade febril materna (indice térmico), presença de corioamnionite e/ ou endometrite. Os parâmetros neonatais avaliados foram o indice de Apgar ( $1^{\circ}$ e $5^{\circ}$ minutos), colonização bacteriana do conduto auditivo e hemocultura. Para a análise estatistica foram utilizados os testes: exato de Fisher, Wilcoxon e o $\chi^{2}$.

Resultados: o uso da ampicilina não prolongou a gestação, não reduziu a morbidade febril puerperal e nem as taxas de corioamnionite e/ ou endometrite. Quanto à morbidade infecciosa perinatal também não foi possivel demonstrar nenhuma redução decorrente do uso da ampicilina nem influência sobre as condições de nascimento. Estes dados foram consistentes em casos de RPM com até 72 horas de evolução, pois o limitado número de casos com tempo maior de evolução não permitiu uma análise estatística isenta de erro tipo II.

Conclusões: com base nos resultados desse trabalho foi possivel concluir que o uso profilático de ampicilina em gestantes com RPM com até 72 horas de evolução não reduz a morbidade infecciosa materna nem perinatal. No entanto, a presença de Streptococcus agalactiae do grupo B em hemocultura de recém-nascido do grupo controle indicou a necessidade imperiosa de antibioticoterapia para as gestantes colonizadas por esse microrganismo.
\end{abstract}

PALAVRAS-CHAVE: Rotura prematura das membranas. Antibioticoprofilaxia. Infecção puerperal.

\section{Introdução}

Em qualquer período da gestação a rotura prematura das membranas (RPM) constitui

Hospital das Clínicas da Faculdade de Medicina de Ribeirão Preto - Universidade de São Paulo.

Correspondência:

Geraldo Duarte

Departamento de Ginecologia e Obstetrícia da Faculdade de Medicina de Ribeirão Preto, Universidade de São Paulo.

Avenida Bandeirantes 3900

14049-900 - Ribeirão Preto - SP preocupação adicional para o tocoginecologista, pois carreia em seu contexto inúmeras controvérsias e indefinições quanto ao seu manuseio e prognóstico. A utilização profilática de antibióticos nessas pacientes faz parte desse grupo de discordâncias e está longe de representar a unanimidade, tanto na rotura prematura das membranas pré-termo (RPM-PT) como naquela que ocorre em gestantes de termo ${ }^{21}$.

Dentre as complicações maternas decorrentes da RPM que poderiam ser reduzidas 
pela antibioticoprofilaxia destacam-se a morbidade febril, corioamnionite e a endometrite puerperal ${ }^{23}$. Por sua vez, dentre as complicações perinatais que potencialmente também poderiam ser reduzidas com antibioticoprofilaxia, sobressaem a prematuridade e a septicemia neonatal ${ }^{14,25}$. Entretanto, as pesquisas sobre o papel da profilaxia antimicrobiana para essas pacientes apresentam resultados discordantes ${ }^{18,22}$.

A infecção genital prévia tem sido considerada importante fator de risco para as complicações infecciosas maternas e perinatais da RPM, principalmente nos casos de gestantes colonizadas por Streptococcus agalactiae - grupo B de Lancefield (SGB) e Escherichia coli ${ }^{25}$.

Apesar de se comprovar o potencial antibacteriano do líquido, sabe-se que essa atividade antimicrobiana é limitada, dependendo da idade gestacional, concentração do complexo zinco-protéico neste fluído e da presença de mecônio. Adicionalmente, tanto a sensibilidade como a resistência de determinados grupos bacterianos ao líquido amniótico também devem ser consideradas. Como exemplo, os germes anaeróbios apresentam elevada sensibilidade aos compostos antibacterianos desse fluído ${ }^{7}$, o que contrasta com a resistência do $\mathrm{SGB}^{6}$. Estes dados subsidiam os achados de colonização bacteriana da câmara amniótica com membranas íntegras, caracterizando a presença de infecção subclínica durante a gestação e que também seriam responsáveis pela amniorrexe em alguns casos ${ }^{12}$. Analisados de forma superficial, esses fatos fornecem argumentos favoráveis ao uso de antibióticos nos casos de amniorrexe, mas quando analisados de forma mais aprofundada, apontam para a necessidade de avaliação mais ampliada, devendo considerar previamente uma série de variáveis em populações específicas ${ }^{11,22}$. Dentre essas variáveis populacionais, a frequência do SGB, a relação custo/benefício da identificação e tratamento das principais infecções genitais no prénatal e o estudo do efeito da antibioticoprofilaxia para gestantes com RPM adquirem caráter prioritário $^{2,5,25}$.

Dentro do contexto da antibioticoprofilaxia em casos de RPM, a literatura é pródiga em resultados discrepantes. Utilizando ensaios randomizados, alguns estudos concluíram que o uso profilático de antimicrobianos para pacientes com RPM pode reduzir a morbidade infecciosa materna quando comparados com o grupo controle $^{9,17}$, enquanto outros não conseguiram demonstrar essa redução $0^{3,8,10}$. Similarmente, não existe unanimidade quanto à morbidade perinatal, havendo resultados favoráveis à antibioticoprofilaxia ${ }^{11,18}$ e outros, segundo os quais essa medida profilática não se mostrou totalmente efetiva $^{16}$, principalmente quando associada a corticoterapia para acelerar a maturidade pulmonar fetal ${ }^{14}$. De forma geral, os resultados perinatais com o uso de antibioticoprofilaxia são melhores em gestantes colonizadas pelo SGB, mas quando se estabelece a mortalidade perinatal com parâmetro, mesmo com aumento do tempo de latência os antibióticos não modificam o resultado final ${ }^{11}$. Outra grande dificuldade com que se deparam os pesquisadores é a reduzida quantidade de estudos controlados e a falta de uniformidade quanto à metodologia utilizada. Esse fato dificulta e frequentemente impede comparações globais entre os resultados obtidos ${ }^{15,16,18,22}$.

Para avaliar a morbidade febril em obstetrícia, muitas vezes a aferição simples da temperatura axilar e a presença de endometrite ou deiscências incisionais não são os melhores parâmetros. Nesse caso, o índice térmico (IT), método descrito por Ledger \& Kriewall ${ }^{13}$, é uma boa alternativa para o estudo da morbidade febril, utilizando programa computacional para o cálculo da área sob a curva térmica.

Tendo em vista a discordância entre os resultados e a grande diversidade dos esquemas propostos para antibioticoprofilaxia em gestantes com RPM, nossa hipótese foi de que o uso profilático de ampicilina, até então empregada no Hospital das Clínicas da Faculdade de Medicina de Ribeirão Preto da Universidade de São Paulo (HCFMRPUSP), não seria necessária para essas pacientes. Para testar essa hipótese, foi proposto avaliar prospectivamente o papel da ampicilina na redução de complicações infecciosas maternas e perinatais decorrentes da RPM.

\section{Pacientes e Métodos}

Foram avaliadas prospectivamente 121 gestantes portadoras de RPM, atendidas no HCFMRP-USP. A seleção de pacientes obedeceu aos seguintes critérios de inclusão: idade gestacional compreendida entre 22 e 41 semanas e 6 dias; RPM com tempo superior a seis horas; serem consideradas gestantes clínica e obstetricamente normais (excetuando a RPM); apresentação fetal cefálica e concordância em participar do estudo. Esta concordância era firmada em formulário previamente aprovado pela Comissão de Ética em Pesquisa do HCFMRP-USP. Qualquer condição 
contrária aos critérios de inclusão bem como o uso prévio de corticóides, antibióticos e/ou substâncias tocolíticas levava à exclusão da paciente do estudo.

O diagnóstico de amniorrexe obedeceu à rotina do Serviço de Gestação de Alto Risco do HCFMRP-USP ${ }^{21}$, incluindo critérios clínicos (anamnese e exame físico) e exames subsidiários, como o teste da cristalização "fern test", aquecimento do contudo cervical em lâmina de vidro e a medida do $\mathrm{pH}$ vaginal. A ecografia foi utilizada para pesquisar oligoidramnia grave, achado que reforçou o diagnóstico de amniorrexe em casos de dúvidas diagnósticas. Além disso, com o exame ecográfico foi possivel avaliar a localização placentária, a presença de bolsões de líquido amniótico, a apresentação e desenvolvimento somático fetal. Algumas pacientes foram excluídas do estudo neste momento, visto serem portadoras de alterações que poderiam dificultar a análise dos dados futuramente.

As pacientes que preencheram os critérios citados anteriormente foram randomizadas em dois grupos. O grupo que recebeu ampicilina por via oral ( $2 \mathrm{~g} / \mathrm{dia})$, por um periodo de 10 dias foi chamado grupo tratado e era composto por 61 pacientes. O grupo que recebeu placebo nas mesmas condições (via de administração, horário e embalagem) foi chamado grupo controle, sendo composto por 60 pacientes. A lista de randomização e as embalagens contendo ampicilina ou placebo foram previamente preparadas e codificadas pela Farmácia do HCFMRP-USP. Salienta-se que nenhum dos pesquisadores tinha conhecimento do conteúdo real das embalagens. Nos casos em que as gestações foram resolvidas por cesárea, a ampicilina ou placebo foram substituídos pela cefalotina (2 gramas após clampar o funículo e 1 grama 6 e 12 horas depois), obedecendo à normatização vigente.

A idade gestacional foi calculada em semanas, utilizando-se a data da última menstruação e, nos casos nos quais a paciente não sabia informar a data, o cálculo foi realizado utilizando-se a ecografia. Com base nestes parâmetros, selecionoaram-se 33 pacientes com menos de 37 semanas de gestação (18 no grupo tratado e 15 no grupo controle) e 88 acima desta idade gestacional ( 44 em cada grupo).

As pacientes foram avaliadas diariamente durante o período anteparto, por meio de critérios clínicos e exames subsidiários. O exame clínico foi realizado com a finalidade de afastar a presença de processos infecciosos localizados e/ou sistêmicos. A temperatura axilar foi medida com intervalos de 4 horas, período necessário para o cálculo do IT, segundo os critérios de Ledger e Kriewall ${ }^{13}$. Os cálculos matemáticos do IT foram realizados utilizando sistema computacional construído pelo setor de computação da FMRPUSP, especialmente para essa finalidade.

O exame laboratorial de controle para o diagnóstico precoce da infecção materna foi o leucograma. Para a avaliação do bem-estar fetal, utilizou-se a ausculta cardiaca fetal com o sonar Doppler e/ou a cardiotocografia anteparto utilizando estímulo acústico, com intervalos de 48 horas. Neste grupo de pacientes evitaram-se toques ou manobras semiológicas vaginais na tentativa de reduzir qualquer risco adicional de corioamnionite ${ }^{24}$. O rigoroso acompanhamento materno anteparto visou o diagnóstico precoce de corioamnionite. Os critérios para o diagnóstico de corioamnionite basearam-se na presença de pelo menos dois dos seguintes sinais/sintomas: temperatura axilar superior a $38^{\circ} \mathrm{C}$, taquicardia materna (>100 bpm), taquicardia fetal (>160 bpm), líquido amniótico purulento e/ou fétido, amolecimento uterino e contagem de leucócitos superior a $15.000 / \mathrm{cm}^{3}$, com desvio à esquerda. A confirmação da corioamnionite determinava o tratamento da infecção, utilizando-se antimicrobianos de amplo espectro que posteriormente eram ajustados, segundo resultado da cultura com antibiograma, complementado-se com resolução da gravidez (parto vaginal ou cesárea), segundo protocolo vigente no HCFMRPUSP. Após a resolução da gravidez as puérperas incluídas no presente estudo foram acompanhadas durante o tempo em que permaneceram internadas no hospital, sendo avaliadas diariamente por meio da análise da curva térmica, tamanho, consistência e sensibilidade uterina, característica dos lóquios e observação do aspecto da cicatriz da episiotomia ou da cesárea. No momento da alta hospitalar as pacientes eram orientadas e recebiam o "medicamento" restante (ampicilina ou placebo) para uso em casa até completar dez dias. Ao término dos dez dias as pacientes retornaram ao hospital para uma avaliação final, ocasião em que foi realizado anamnese, exame clínico e exame ginecológico, pesquisando-se infecção ligada ao parto e outros sinais/sintomas que indicassem necessidade de cuidados médicos. As puérperas que não retornaram no período de 10 a 15 dias foram visitadas em seus domicílios pelos pesquisadores envolvidos.

A análise estatística foi realizada usando os seguintes testes não-paramétricos: $\chi^{2}$, exato de Fisher e Wilcoxon, com nivel de significância de $5 \%(\mathrm{p}<0.05)$. 
Resultados

Do total de pacientes randomizadas, 121 gestantes portadoras de PRM e seus recémnascidos (RN) tiveram o seguimento completo e constituíram a casuística deste estudo, sendo 61 no grupo que recebeu ampicilina e 60 no grupo que recebeu placebo.

Os dois grupos foram semelhantes quanto ao número de pacientes, idade, cor, paridade, número de abortamentos prévios, tempo de rotura das membranas por ocasião da admissão no hospital, idade gestacional e tempo compreendido entre a RPM e a resolução da gravidez (Tabela 1).

Tabela 1 - Características das pacientes com rotura prematura de membranas.

\begin{tabular}{lcc}
\hline Parâmetros & Grupo tratado & Grupo controle \\
\hline $\mathrm{N}$ & 61 & 60 \\
Idade materna & $25,7 \pm 6,8$ & $27,1 \pm 6,8$ \\
& & $47(78,3 \%)$ \\
Brancas & $42(68,8 \%)$ & $13(21,7 \%)$ \\
Negras & $19(31,2 \%)$ & $16(26,7 \%)$ \\
& & $37(61,7 \%)$ \\
Nulíparas & $27(44,3 \%)$ & $7(11,6 \%)$ \\
1-4 filhos & $28(45,9 \%)$ & $48(80,0 \%)$ \\
$>4$ filhos & $6(9,8 \%)$ & $12(20,0 \%)$ \\
Nenhum aborto prévio & $43(70,5 \%)$ & $14,0 \pm 9,5$ \\
1-3 abortos prévios & $18(29,4 \%)$ & $39,3 \pm 31,4$ \\
& & $37,9 \pm 2,9$ \\
\hline
\end{tabular}

Na Tabela 2 encontram-se os dados referentes à morbidade infecciosa materna, observando-se que 57 pacientes do grupo tratado $(93,5 \%)$ e 57 do grupo controle $(95,0 \%)$ não apresentaram quaisquer manifestações infecciosas. A corioamnionite foi detectada em 3 pacientes do grupo tratado $(4,9 \%)$ e em 2 pacientes do grupo controle $(3,3 \%)$. Uma paciente em cada grupo $(1,6 \%)$, além da corioamnionite, também evoluiu para endometrite. A análise estatística desses dados não demonstrou nenhuma diferença significativa. O IT obtido também não detectou nenhuma diferença estatística entre os dois grupos.

Tabela 2 - Presença de infecções maternas e de morbidade febril (índice térmico).

\begin{tabular}{lccc}
\hline $\begin{array}{l}\text { Parâmetros de } \\
\text { infecção }\end{array}$ & Grupo tratado & Grupo controle & Significância \\
\hline Ausentes & $57(93,5 \%)$ & $57(95,0 \%)$ & Não \\
Corioamnionite & $3(4,9 \%)$ & $2(3,3 \%)$ & Não \\
Corioamnionite + & $1(1,6 \%)$ & $1(1,7 \%)$ & Não \\
endometrite & & & \\
Índice térmico $\left({ }^{\circ} \mathrm{C} /\right.$ hora) & $3,0 \pm 0,9$ & $2,6 \pm 0,8$ & Não \\
\hline
\end{tabular}

Na Tabela 3 encontram-se os dados sobre o tipo de parto e sua correlação com a morbidade infecciosa materna. Sobre o tipo de parto realizado, observa-se que a resolução na maioria das pacientes do grupo tratado $(53=86,9 \%)$ e do grupo controle $(53=88,4 \%)$ ocorreu pela via transvaginal. O número total de cesáreas também foi estatisticamente semelhante nos dois grupos, observando-se 8 casos (13\%) no grupo tratado e 7 casos $(12,6 \%)$ no grupo controle, não havendo nenhuma influência do uso da ampicilina na redução da taxa de cesárea neste grupo de pacientes.

O número total de diagnóstico de infecções uterinas entre as 53 pacientes do grupo tratado que tiveram parto transvaginal foi 3 ( 3 casos de corioamnionite), representando $5,7 \%$ neste subgrupo de pacientes. Entre as 53 pacientes do grupo controle cujas gestações foram resolvidas por meio de parto vaginal, nenhuma desenvolveu infecção intra-uterina. Avaliando as 8 pacientes do grupo tratado que foram submetidas à cesárea, verificou-se que 2 delas apresentaram infecção intra-uterina (um caso de corioamnionite e um caso de corio + endometrite), representando $25 \%$ 
neste subgrupo de pacientes. Analisando essa mesma situação entre as 7 pacientes do grupo controle submetidas à cesárea, verificaram-se 3 casos de infecção intra-uterina ( 2 casos de corioamnionite e um caso de corio + endometrite), representando $42,8 \%$. Apesar da diferença percentual o teste exato de Fisher não mostrou nenhuma diferença estatística $(p=0,42)$. Nos dois grupos estas infecções ocorreram em gestantes cujo tempo de latência ultrapassou 72 horas e o volume amniótico avaliado pela ecografia revelou ausência desse fluído.

Tabela 3 - Tipo de parto e morbidade infecciosa materna

\begin{tabular}{lccc}
\hline Parâmetros & Grupo tratado & Grupo controle & Significância \\
\hline Parto vaginal (infecção ausente) & $51(83,6 \%)$ & $53(87,4 \%)$ & Não \\
Parto vaginal (corioamnionite) & $2(3,3 \%)$ & 0 & - \\
Cesárea (infecção ausente) & $6(9,8 \%)$ & $4(6,7 \%)$ & Não \\
Cesárea (corioamnionite) & $1(1,6 \%)$ & $2(3,3 \%)$ & Não \\
Cesárea (corioamnionite + endometrite) & $1(1,6 \%)$ & $1(1,6 \%)$ & Não \\
\hline
\end{tabular}

Os resultados neonatais estão distribuídos na Tabela 4, verificando-se que não houve diferenças significativas entre o grupo tratado e o grupo controle quanto aos seguintes parâmetros: peso ao nascer $(2.858,2 \pm 709,9$ versus $2.851,8 \pm$ 662,5 gramas respectivamente), estatura ao nascer $(48,1 \pm 3,4$ versus $47,5 \pm 3,1$ centímetros respectivamente), índice de Apgar no $1^{\circ}$ minuto $(7,5$ $\pm 2,7$ versus $8,0 \pm 2,1$, respectivamente), indice de Apgar no $5^{\circ}$ minuto $(9,2 \pm 1,5$ versus $9,4 \pm 1,3$, respectivamente). Houve um caso de natimorto no grupo tratado $(1,5 \%)$ e nenhum no grupo controle. Morte neonatal precoce ocorreu em 2 neonatos de cada grupo $(3,2 \%$ e $3,3 \%$ respectivamente). A análise desses dados não mostrou nenhuma diferença estatística.

Tabela 4 - Resultado perinatal

\begin{tabular}{lccc}
\hline Parâmetros & Grupo tratado & Grupo controle & Significância \\
\hline$N^{0}$ de recém-nascidos* & 64 & 61 & \\
Peso $(\mathrm{g})$ & $2858,2 \pm 709,9$ & $2851,8 \pm 662,5$ & Não \\
Comprimento $(\mathrm{cm})$ & $48,1 \pm 3,4$ & $47,5 \pm 3,1$ & Não \\
& & & \\
Indices de Apgar & & & \\
1 min & $7,5 \pm 2,4$ & $8,0 \pm 2,1$ & Não \\
5 min & $9,2 \pm 1,5$ & $9,4 \pm 1,3$ & Não \\
Morte fetal & $1(1,5 \%)$ & 0 & - \\
Morte neonatal & $2(3,2 \%)$ & $2(3,3 \%)$ & Não \\
\hline
\end{tabular}

Quanto à morbidade infecciosa neonatal, a leucocitose esteve presente em $4,5 \%$ dos neonatos do grupo tratado e em $1,6 \%$ do grupo controle, mas esta diferença não se mostrou significativa. É interessante ressaltar que a maioria absoluta de todos os RN não apresentaram nenhuma alteração infecciosa do leucograma.

A cultura de material colhido do conduto auditivo dos RN mostraram-se positivas em 13 neonatos do grupo tratado $(20,3 \%)$ e em 12 do grupo controle $(19,7 \%)$. Os microrganismos isolados de material colhido do conduto auditivo dos RN do grupo tratado foram: Escherichia coli (10 casos), Staphylococcus epidermidis (2 casos) e Klebsiella pneumoniae (1 caso), ao passo que nos neonatos do grupo controle detectou-se colonização por Escherichia coli (6 casos), Staphylococcus epidermidis (2 casos), Streptococcus grupo D de Lancefield - não-enterococo (1 caso), SGB (1 caso), Streptococcus pneumoniae (1 caso) e Streptococcus viridans (1 caso).

Constatou-se que o microrganismo mais freqüentemente observado entre os RN com cultura positiva dos dois grupos foi a Escherichia coli, com $76,9 \%$ no grupo que recebeu ampicilina e $50 \%$ no grupo controle. Observou-se que os $12 \mathrm{RN}$ de mães do grupo controle foram colonizados no conduto auditivo por 6 tipos diferentes de microrganismos, ao passo que $13 \mathrm{RN}$ do grupo tratado foram colonizados por apenas 3 tipos diferentes de microrganismos.

A hemocultura foi positiva em $6(9,7 \%)$ e 8 $(13,1 \%)$ neonatos dos grupos tratado e controle, respectivamente. A análise desses números pelo teste do $\chi^{2}$ não mostrou nenhuma diferença estatística ( $\mathrm{p}=0,7)$. Os microrganismos isolados no grupo tratado foram a Escherichia coli (4 casos) e a Pseudomonas sp (2 casos), enquanto que no grupo controle foram a Pseudomonas sp (2 casos), Bacteroides sp, Clostridium sp, Corynebacterium sp, SGB, Streptococcus D (não-enterococo) e 
Staphylococcus epidermidis (todos com 1 caso cada um). O microrganismo isolado mais freqüentemente no grupo tratado foi a Escherichia coli $(66,7 \%)$, ao passo que no grupo que recebeu placebo foi Pseudomonas sp (25\%).

\section{Discussão}

A conduta expectante diante dos casos de RPM visa o prolongamento da gravidez para se obterem melhores condições de maturidade pulmonar fetal. Entretanto, a manutenção da gravidez, mesmo que por alguns dias ou até mesmo algumas horas, expõe a cavidade amniótica à ação dos microrganismos da flora vaginal, estabelecendo um definido jogo entre os beneficios e os potenciais danos maternos e/ou perinatais decorrentes da conduta adotada. Com base nesses fatos a antibioticoprofilaxia tem sido proposta por ocasião da conduta expectante com o propósito de reduzir a flora infectante, permitindo que a gestação se prolongue $^{1}$. No entanto, há controvérsias sobre o beneficio global dessa conduta.

Considerando o uso profilático de ampicilina em casos de RPM visando o prolongamento da gestação, nessa casuística constatou-se que não houve nenhum beneficio neste sentido. O tempo médio decorrido entre a ruptura das membranas ovulares e a resolução da gravidez foi semelhante entre as gestantes do grupo tratado e aquelas do grupo controle. Entre gestantes com PRM colonizadas com SGB, Mercer et al. ${ }^{18}$ encontraram resultados semelhantes.

Considerando a morbidade febril materna em pacientes com RPM, o presente estudo mostrou que a antibioticoprofilaxia não a modificou, visto que o IT foi praticamente igual nos dois grupos. $\mathrm{O}$ IT mede indiretamente a quantidade de temperatura por tempo e constitui um bom indicador da morbidade febril, que em última análise se relaciona diretamente com presença de febre e tempo de permanência hospitalar ${ }^{13}$. Indiretamente, elevados valores do IT se relacionam com a presença de complicações infecciosas de resolução demorada, geralmente elevando os custos hospitalares. Neste quesito, os dados desse estudo não demonstram nenhuma diferença com o uso profilático de ampicilina em casos de PA. Este dado está intimamente ligado ao fato de a incidência de cesárea não ter sido afetada pelo uso da antibioticoprofilaxia, concordando com os achados de Mercer e Arheart ${ }^{17}$. Como se sabe, a cesárea nesses casos apresenta uma relação direta com o aumento da morbidade febril puerperal ${ }^{24}$.

Observou-se que a infecção intra-uterina em gestantes com RPM complicou $6,5 \%$ das pacientes do grupo tratado e $5,0 \%$ daquelas que compuseram o grupo controle, taxa que não foi modificada pelo uso profilático de ampicilina. A abordagem terapêutica visando o controle dessas complicações seguiu as normas protocolares vigentes no HCFMRP-USP, utilizando antimicrobiano diferente daquele utilizado com finalidade profilática. Se por um lado os resultados aqui obtidos não confirmaram os achados de algumas casuísticas ${ }^{9,17}$, por sua vez confirmam os resultados de outros autores ${ }^{3,8,10,16,20}$, segundo os quais a antibioticoprofilaxia em casos de amniorrexe não proporciona beneficios maternos adicionais quando comparada à administração de placebo.

A análise da correlação entre o tipo de resolução da gravidez e as complicações infecciosas intra-uterinas não mostrou diferenças entre o grupo tratado com ampicilina e aquele que recebeu placebo, considerando as duas formas de resolução da gravidez. Apesar de ser considerada um fator adicional no aparecimento da complicação infecciosa em casos de RPM, deve ser considerado na presente casuística que a cesárea foi utilizada com maior freqüência para a resolução de casos em que a indução do trabalho de parto não obteve sucesso ou havia urgência para a resolução da gravidez. As 2 pacientes complicadas por endometrite puerperal tinham diagnóstico prévio de corioamnionite, e seus fetos apresentaram sinais de comprometimento durante o trabalho de parto. Entretanto observou-se que, isoladamente, a cesárea mostrou ser uma condição que predispõe à morbidade infecciosa quando comparada ao parto vaginal. Apesar de não haver diferença estatisticamente significativa dessa forma de resolução da gravidez entre os dois grupos, devese considerar que o pequeno número de amostras permite a ocorrência de erro estatístico tipo II, indicando a necessidade de ampliar a casuística.

Constatou-se que o microrganismo mais freqüentemente isolado no conduto auditivo dos RN dos dois grupos foi a Escherichia coli $(76,9 \%$ no grupo tratado e $50 \%$ no grupo controle). A colonização por um maior número de grupos bacterianos no grupo controle que no grupo tratado parece estar ligada ao espectro de ação antimicrobiano da ampicilina, eliminando os microrganismos sensiveis a este antibiótico, explicando assim o menor número de grupos bacterianos isolados neste grupo. Como a Escherichia coli é um dos mais prevalentes microrganismos presentes nas infecções neonatais 
decorrentes de RPM, a redução que se obteve com a ampicilina não correspondeu às expectativas.

Apesar de não haver diferença significativa entre o número de hemoculturas positivas entre os RN dos dois grupos desse estudo, constatamos que no grupo sem antibioticoprofilaxia foi observado um caso de septicemia pelo $\mathrm{SGB}$, o que gerou grande preocupação, fazendo com que a cultura genital desse microrganismo fosse incorporada ao grupo de medidas que visam o controle de gestantes com RPM no HCFMRP-USP. Melhor seria ainda se houvesse condições de cultivo desse microrganismo durante a gestação.

No tocante ao efeito da antibioticoprofilaxia em gestantes com RPM para evitar a morbidade infecciosa perinatal geral, os dados dessa casuística estão de acordo com alguns resultados divulgados na literatura ${ }^{16,19,22}$. No entanto, são diferentes dos resultados obtidos por Mercer et al. ${ }^{18}$. Esses autores estudaram 614 gestantes portadoras de RPM-PT e seus RN, mostrando que a antibioticoprofilaxia antenatal reduziu a morbidade infecciosa perinatal. Apesar de ter utilizado um discutivel esquema antimicrobiano (ampicilina e eritromicina por via endovenosa durante 48 horas, seguido de amoxicilina e eritromicina, via oral, por 5 dias adicionais), esses dados influenciaram o American College of Obstetricians and Gynecologists $(\mathrm{ACOG})^{1}$ na inclusão dessa conduta no rol de medidas utilizadas no controle de gestantes com RPM-PT. O argumento principal para a adoção da antibioticoprofilaxia é a erradicação de bactérias normalmente presentes na genitália feminina, notadamente o $\mathrm{SGB}^{2,4,5,6}$. Estranhamente, justo no grupo de gestantes portadoras do SGB, a antibioticoprofilaxia utilizada não conseguiu aumentar o período de latência, que foi igual ao do grupo que utilizou placebo. Outra variável que pode explicar a diferença entre os dados de Mercer et al. ${ }^{18}$ e os da presente casuística é o número de gestantes com RPM-PT. A ausência de diferença significante talvez se deva ao pequeno número de pacientes avaliadas neste trabalho não tenha poder de discriminação suficiente para demonstrar maior clareza da necessidade de antibioticoprofilaxia, principalmente nos casos com mais de 72 horas de evolução.

O único caso de óbito fetal observado nessa casuística ocorreu em feto do grupo tratado. Sua necrópsia não constatou infecção e o óbito teve como causa básica a anóxia intra-uterina, associada à prematuridade. Os óbitos neonatais, entretanto, associaram prematuridade extrema e presença de infecção que a ampicilina não conseguiu evitar.
Em relação à morbidade e mortalidade por causas infecciosas em casos de RPM, com base nos resultados aqui obtidos pode-se afirmar que a utilização profilática da ampicilina em gestantes com até 72 horas de latência não beneficiou mães ou seus RN. O fato de os RN do grupo controle serem colonizados por uma população mais variada de microrganismos (demonstrado laboratorialmente) não se manifestou clinicamente. Com base nestes dados, o cuidado para a gestante com RPM no HCFMRP-USP não inclui antibioticoprofilaxia. Entretanto, reconhecendo a limitação numérica da presente casuística, vislumbra-se a necessidade de novos estudos com casuísticas ampliadas e talvez diferentes antimicrobianos, principalmente entre aquelas gestantes com período de latência prolongado.

\section{SUMMARY}

Purpose: to evaluate whether prophylactic use of ampicillin could avoid or reduce maternal and perinatal infectious morbidity caused by premature rupture of membranes (PROM), and to extend the gestation period in those women. Methods: this was a prospective, randomized and double-blind study, carried out evaluating 121 pregnant women with PROM, randomized into two study groups. The treatment group (61 patients) received ampicillin and the control group (60 patients) received placebo. The placebo had the same characteristics as ampicillin (kind of packaging and color of the capsules) and was used in the same time regimen. The considered parameters for maternal infection were febrile morbidity (fever index), and the presence of chorioamnionitis and/or endometritis. The studied neonatal parameters were Apgar score (1st and 5th minutes), bacterial colonization of auditory canal, and blood culture. The statistical tests performed were Fisher's exact test, Wilcoxon, and $\chi^{2}$.

Results: it was observed that ampicillin did not prolong the gestation, nor did it reduce the postpartum febrile morbidity or the rates of chorioamnionitis and/or endometritis. Ampicillin did not reduce the perinatal infectious morbidity nor improve the birth outcomes. All these results were consistent in cases of less than $72 \mathrm{~h}$ PROM. The limited number of cases with time of PROM greater than $72 \mathrm{~h}$ did not permit statistical analysis free of type II error.

Conclusions: based on these results it was possible to conclude that the prophylactic use of ampicillin by pregnant women with less than 72 h PROM did not reduce either infectious maternal or perinatal morbidity. However, the presence of group $B$ Streptococcus agalactiae in the blood culture from a neonate in the control group showed the necessity to start antibiotic treatment of pregnant women colonized by this microorganism.

KEY WORDS: Premature rupture of membranes. Antibiotic prophylaxis. Postpartum febrile morbidity. 


\section{Referências}

1. American College of Obstetricians and Gynecologists (ACOG). Premature rupture of membranes. Int $\mathrm{J}$ Gynecol Obstet 1998; 63: 75-84.

2. American College of Obstetricians and Gynecologists (ACOG). Prevention of early-onset group B streptococcal disease in newborns. ACOG Comm Opin 1996; 173: 1-8.

3. Amon E, Lewis SV, Sibai BM, Villar MA, Arheart KL. Ampicillin prophylaxis in preterm premature rupture of the membranes: A prospective randomized study. Am J Obstet Gynecol 1988;159: 539-43.

4. Bramer S; van Wijk FH, Mol BW, Adriaanse AH. Risk indicators for neonatal early-onset GBS-related disease. A case-control study. J Perinat Med 1997; 25: 469-75.

5. Cararach V, Botet F, Sentis J, Almiral R, Perez PE. Administration of antibiotics to patients with rupture of membranes at term: a prospective, randomized, multicentric study. Collaborative Group on PROM. Acta Obstet Gynecol Scand 1998; 77: 298-302.

6. Centers for Disease Control and Prevention (CDC): Prevention of perinatal group B streptococcal disease: a public health perspective. MMWR 1996; 45(RR-7): 1-24.

7. Duarte G. Efeitos da diluição, aquecimento e esterilização do líquido amniótico sobre sua atividade antibacteriana contra o Bacteroides fragilis. Tese (Doutorado). Departamento de Ginecologia e Obstetrícia da Faculdade de Medicina de Ribeirão Preto, Universidade de São Paulo; 1988.

8. Egarter C, Leitich H, Karas H, Wieser F, Husslein P, Kaider A, et al. Antibiotic treatment in preterm premature rupture of membranes and neonatal morbidity: A metaanalysis. Am J Obstet Gynecol 1996, 174: 589-97.

9. Ernest JM, Givner LB. A prospective randomized placebo-control trial of penicillin in preterm premature rupture of membranes. Am J Obstet Gynecol 1994; 170: 516-21.

10.Gareth SP, Hannah ME, Myhr TL, Farine D, Ohlsson A, Wang EE, et al. International Multicentre Term Prelabor Rupture of Membranes Study: Evaluation of predictors of clinical chorioamnionitis and postpartum fever in patients with prelabor rupture of membranes at term. Am J Obstet Gynecol 1997; 177: 1024-9.

11.Gibbs RS, Eschenbach DA. Use of antibiotics to prevent preterm birth. Am J Obstet Gynecol 1997; 177: 37580.

12.Gomez R, Romero R, Galasso M, Behnke E, Insunza A, Cotton DB. The value of amniotic fluid interleukin-6, white blood cell count, and Gram stain in the diagnosis of microbial invasion of the amniotic cavity in patients at term. Am J Reprod Immunol 1994; 32: 200-10.
13.Ledger WJ, Kriewall TJ. The fever index: a quantitative indirect measure of hospital-acquired infections in obstetrics and gynecology. Am J Obstet Gynecol 1993; 115: 514-20.

14.Leitich H, Egarter C, Reisemberg K, Kaider A, Berghammer P. Concomitant use of glucocorticoids: A comparison of two metaanalyses on antibiotic treatment in preterm premature rupture of membranes. Am J Obstet Gynecol 1998; 178: 899908.

15.Lovet SM, Weiss JD, Diogo MJ, Williams PT, Garite TJ. A prospective double-blind, randomized, controlled clinical trial of ampicillin-sulbactam for preterm premature rupture of membranes in women receiving antenatal corticosteroid therapy. Am J Obstet Gynecol 1997; 176: 1030-8.

16.McGregor JA, French JI, Seo K. Antimicrobial therapy in preterm premature rupture of membranes: Results of a prospective, double-blind, placebocontroled trial of erythromycin. Am J Obstet Gynecol 1991; 165: 632-40.

17.Mercer BM, Arheart KL. Antimicrobial therapy in expectant management of preterm premature rupture of the membranes. Lancet 1995; 346: 1271 9.

18.Mercer BM, Miodovnik M, Thurnau GR, Goldenberg RL, Dias AF, Ramsey RD, et al. Antibiotic therapy for reduction of infant morbidity after preterm premature rupture of the membranes. A randomized controlled trial. JAMA 1997; 278: 989 95.

19. Mercer BM, Moretti ML, Prevost RR, Sibai BM. Erythromycin therapy in preterm premature rupture of the membranes: A prospective, randomized trial of 220 patients. Am J Obstet Gynecol 1992; 166: 794-802.

20.Morales WJ, Angel JL, O’Brien WF, Knuppel RA. Use of ampicillin and corticosteroids in premature rupture of membranes: A prospective randomized study. Obstet Gynecol 1989; 73: 721-6.

21. Nogueira AA, Duarte G, Cunha SP. Amniorrexe prematura. In: Cunha SP, Duarte G, editores. Gestação de Alto Risco. São Paulo: MEDSI; 1998. p. 441-47.

22. Ohlsson A. Treatment of preterm premature rupture of the membranes: A meta-analysis. Am J Obstet Gynecol 1989; 160: 890-906.

23.Romero R, Ghidini A, Mazor M, Behnke E. Microbial invasion of the amniotic cavity in premature rupture of membranes. Clinical Obstet Gynecol 1991; 34: 769-78.

24.Soper DE, Mayhall CG, Froggatt JW. Characterization and control of intraamniotic infection in an urban teaching hospital. Am J Obstet Gynecol 1996; 175: 304-9.

25. Sweet RL, Gibbs RS. Premature rupture of membranes. In: Sweet RL, Gibbs RS, editores. Infectious Diseases of the Female Genital Tract. Baltimore: Williams \& Wilkins; 1995. p. 529-47. 